\title{
Cathepsin $L$ interacts with CDK2-AP1 as a potential predictor of prognosis in patients with breast cancer
}

\author{
ZHAN WANG $^{1 *}$, ZHEN XIANG $^{1 *}$, TING ZHU $^{1}$, JUAN CHEN $^{2}$, MEI-ZUO ZHONG ${ }^{1}$, JUAN HUANG $^{3}$, \\ KUAN-SONG WANG ${ }^{4}$, LING LI ${ }^{5}$, LUN-QUAN SUN ${ }^{5}$ and WEI-BING ZHOU ${ }^{1}$ \\ Departments of ${ }^{1}$ Oncology and ${ }^{2}$ Pharmacy, Xiangya Hospital, Central South University; \\ ${ }^{3}$ Hunan Province Clinic Meditech Research Center for Breast Cancer; ${ }^{4}$ Department of Pathology; \\ ${ }^{5}$ Center for Molecular Medicine, Xiangya Hospital, Central South University, Changsha, Hunan 410008, P.R. China
}

Received January 22, 2019; Accepted September 20, 2019

DOI: $10.3892 / \mathrm{ol} .2019 .11067$

\begin{abstract}
Cathepsin L (CTSL) is a lysosomal acid cysteine protease that has been implicated in tumorigenesis and malignant progression. In the present study, the role of CTSL in tumorigenesis and prognosis of breast cancer was evaluated. The prognostic value of CTSL was analyzed using immunohistochemistry in patients with breast cancer, as well as online microarray datasets. CTSL expression was knocked down in the breast cancer cell line T-47D using RNA interference. MTT and colony formation assays were performed to assess the role of CTSL in the proliferation of breast cancer cells. Cell cycle progression and apoptosis were measured using flow cytometry. A physical interaction of CTSL and cyclin dependent kinase 2 associated protein 1 (CDK2-AP1) was determined using a glutathione S-transferase pull-down assay. Endogenous CTSL expression was high in breast cancer cells and exhibited an inverse association with CDK2-AP1 expression; aberrant expression of CTSL in breast cancer tissues predicted an improved clinical outcome and prognosis. In addition, CTSL knockdown decelerated the progression of breast cancer cells by arresting cell cycle progression and increasing apoptosis. Thus, CTSL may be a potential therapeutic target for treating patients with breast cancer.
\end{abstract}

\section{Introduction}

Breast cancer is the most prevalent malignancy in women and estimated to account for $30 \%$ of new cancer diagnoses

Correspondence to: Professor Wei-Bing Zhou, Department of Oncology, Xiangya Hospital, Central South University, 87 Xinagya Road, Changsha, Hunan 410008, P.R. China

E-mail: zhouweibing298@csu.edu.cn

*Contributed equally

Key words: breast cancer, Cathepsin L, cyclin-dependent kinase 2-associated protein 1 , prognosis in women and $15.3 \%$ of all types of cancer according to a statistics report in 2018 in the United States (1). The incidence rate varies greatly worldwide from 19.3 per 100,000 women in Eastern Africa to 89.7 per 100,000 women in Western Europe, and the highest annual morbidity (40,290 cases, in 2015) and mortality rate $(13.8 \%$, in 2015) of patients is in the United States (2,3). Between 2002 and 2008, the mortality rate from breast cancer increased by $201 \%$ among urban Chinese women (4). Triple-negative breast cancer in particular is associated with poor prognosis due to a lack of clinically established targeted therapies and aggressive pathological characteristics (5). Despite advanced systemic treatments including surgical excision, local radiotherapy and adjuvant therapy, which have successfully doubled the survival rate of patients with breast cancer (6), 10-15\% patients still experience recurrence or metastasis (7). Developing an efficient prevention and/or treatment regimen for patients with breast cancer remains a major challenge in clinical practice.

Carcinogenesis is the consequence of the synergistic effects of imbalanced homeostasis and aberrant multigenetic regulation. Accumulating experimental evidence has suggested several molecular hallmarks in breast cancer, such as breast and ovarian cancer susceptibility protein 1 (BRCA1) and BRCA2, which have been used to assess the risk of developing breast cancer (8-10). Cathepsins are ubiquitous proteinases that serve important roles in cancer metastasis and degrade proteins in the lysosome; based on the variation of their active sites, cathepsins can be divided into three subgroups: Aspartate (D and E), Cysteine (B, C, H, F, K, L, O, S, V, W and X/Z), and Serine $(\mathrm{G})$ cathepsins (11). Cathepsin D serves a crucial biomarker in the early detection of nasopharyngeal cancer and metastasis in patients with breast cancer $(12,13)$. Recently, Cathepsin L (CTSL) was demonstrated to be associated with multiple diseases, including breast cancer metastasis (14-17). However, the specific mechanisms and functions of CTSL in breast cancer metastasis are still unclear. Although accumulating evidence has revealed that CTSL expression is increased in several types of carcinoma, including breast cancer, and that its expression pattern reflects the degree of malignancy, the detailed mechanisms and its interaction partners remain elusive (18). 
The aim of the present study was to investigate the expression profiles and pathological functions of CTSL and its contribution to the prognosis of breast cancer, highlighting CTSL as a potential novel therapeutic target for the treatment of patients with breast cancer.

\section{Materials and methods}

Patient selection and follow-up. A total of 249 primary breast cancer tissues and 31 paired adjacent normal tissues were collected from patients admitted to the Xiangya Hospital of Central South University (Changsha, China) between July 2004 and July 2005. The patients enrolled were histologically confirmed as cases of primary breast cancer, underwent surgical resection and had an adequate size tissue sample with no evidence of metastasis prior to the surgery. Patients with previous or other concomitant malignancies were excluded. The clinical information of the patients was collected from Xiangya Hospital, including age, menstrual status, status of estrogen receptor (ER), progesterone receptor (PR) and human epidermal growth factor receptor 2 (HER-2) expression, clinical stage (classified according to the 7 th edition of the American Joint Committee on Cancer staging manual) (19), recurrence and metastasis. The characteristics of the patients are presented in Table I. The termination date for patient follow-up was June 2015. Progression-free survival (PFS) was calculated as the time between the date of resection and death, recurrence or metastasis. Alive patients without recurrence or metastasis at the end of the follow-up were censored. The study was conducted in accordance with the Declaration of Helsinki (20), and the protocol was approved by The Ethical Review Committee of Xiangya Hospital, Central South University.

Immunohistochemistry. Immunohistochemistry was performed as described previously (21). Briefly, paraffin-embedded tissues were cut into $5-\mu \mathrm{m}$ sections and mounted on slides. The slides were deparaffinized by xylene, rehydrated with graded ethanol series and stained with a CTSL antibody (1:200; cat. no. TA809346; OriGene Technologies, Inc.) overnight at $4^{\circ} \mathrm{C}$ and subsequently incubated with a biotinylated secondary goat anti-mouse immunoglobulin $\mathrm{G}$ antibody (1:1,000; cat. no. TA130001; OriGene Technologies, Inc.). The immunoreactions were detected using a streptavidin-peroxidase system. The slides were evaluated in a double-blinded manner by two independent pathologists. The intensity of the staining was scored as follows: 0, negative/-; 1 , weak/+; 2, moderate/++; and 3, strong/+++. The proportion of immunoreactive cells was scored as follows: 0 , no cells stained; $1,<25 \%$ of cells stained; $2,25-50 \%$ of cells stained; 3 , $51-75 \%$ of cells stained; and $4,>75 \%$ of cells stained. The final score for each tissue specimen was determined by multiplying the score for the proportion of immunoreactive cells by the score for the intensity of the staining. In order to ascertain a cut-off score to distinguish between the expression levels of CTSL, receiver operating characteristic (ROC) curve analysis was used. The score closest to the point of maximum Youden's index was used as the optimal cut-off value. CTSL low expression was defined as samples with scores below the cut-off value (score $<6.5$ ), whereas high expression was defined as samples with scores above the value (score $>6.5$ ).
Protein-protein interaction (PPI) network construction. The STRING database (string-db.org; version 10) was searched for cyclin-dependent kinase 2 -associaed protein 1 ('CDK2-AP1') in 'homo sapiens' to identify the potential protein-protein interactions.

Oncomine database. To determine the expression of CTSL in human breast cancer, data mining was performed using the Oncomine database (www.oncomine.org). 'Sorlie breast', 'Sorlie breast 2', 'Perou breast', 'Ma breast 4', 'Zhao breast' and 'Richardson breast 2' databases were selected (22-27). The gene expression of CTSL between cancer tissues and normal tissues was compared. In addition, the expression of CTSL in different histological grades and clinical stages of breast cancer were also determined.

PROGgene database. The prognostic significance of CTSL was assessed using the PROGgene database (genomics.jefferson. edu/proggene/). GSE10893-GPL887, GSE6130-GLP1390 and GSE9893 (28-30) were the datasets used for further analysis. Kaplan-Meier analysis was used to evaluate the prognostic significance of CTSL.

Yeast two-hybrid ( $\mathrm{Y} 2 \mathrm{H})$ screening. Y2H library screening was performed as described in the Matchmaker ${ }^{\circledR}$ Gold Y2H System User Manual (Clontech Laboratories, Inc.) with minimal modifications. Briefly, the pGBKT7-CDK2-AP1 BD-Bait plasmid was generated and transformed into the Y2H Gold Yeast Strain, and the bait expression, autoactivation and cytotoxicity were assessed prior to any application. Subsequently, concentrated Y2H Gold [pGBKT7-CDK2-AP1] recombinants were mixed with $1 \mathrm{ml}$ library strains [Mate\&Plate ${ }^{\mathrm{TM}}$ Libraries-Universal Human (Normalized), Clontech Laboratories, Inc.] and incubated at $30^{\circ} \mathrm{C}$ with gentle agitation until the zygotes were visible under a phase-contrast microscope (CKX41; Olympus Corporation). The desired colonies were screened from the DDO/X/A plates (double synthetic dropout medium lacking tryptophan and leucine supplemented with $\mathrm{X}-\alpha-\mathrm{Gal}$ and Aureobasidin A) dependent on the color, and the plasmids were extracted from the positive colonies, sequenced and analyzed using NCBI BLAST (http://www.ncbi.nlm.nih.gov) and the results were obtained. Then, protein interaction maps were generated using Cytoscape (https://cytoscape.org/) (31) and interaction networks of the 13 putative CDK2-AP1-interacting proteins were examined using Search Tool for the Retrieval of Interacting Genes and Proteins (STRING) (32).

Cell cultures. The human breast cancer cell lines T-47D, MCF-7, MDA-MB-231 and BT-474, as well as 293T cells were purchased from the Type Culture Collection of the Chinese Academy of Sciences; all cell lines were authenticated by American Type Culture Collection. The T-47D, MDA-MB-231 and 293T cells were routinely cultured in DMEM (HyClone; GE Healthcare Life Sciences) supplemented with $10 \%$ fetal bovine serum (FBS; Lonsera Science); the BT-474 cells were cultured in RPMI-1640 medium (HyClone; GE Healthcare Life Sciences) supplemented with $10 \%$ FBS; and the MCF-7 cells were cultured in DMEM supplemented with $10 \%$ FBS, $1 \%$ sodium glutamate and $0.01 \mathrm{mg} / \mathrm{ml}$ bovine insulin. All the cells were incubated at $37^{\circ} \mathrm{C}$ in a humidified atmosphere with $5 \% \mathrm{CO}_{2}$. 
Lentivirus packaging and infection. CTSL small interfering (si)RNAs (s1, 5'-CCAAAGACCGGAGAAACCATT-3'; s2, 5'-AGGCGATGCACAACAGATTAT-3'; and s3, 5'-TGCCTC AGCTACTCTAACATT-3') were designed against the open reading frame of the human CTSL gene (NM_145918.2) and a random non-targeting siRNA (5'-TTCTCCGAACGTGTC ACGT-3') was designed as a negative control. Fragments of the siRNA were cloned into the pGreenPuro $^{\mathrm{TM}}$ short hairpin $(\mathrm{sh})$ RNA Cloning and Expression Lentivector (System Biosciences, LLC) and recombinant lentiviruses were produced by co-infecting 293T cells with the siRNA expression plasmid and pHelper plasmids (Lentiviral Packaging mix; Sigma-Aldrich; Merck KGaA). For lentiviral infection, $1 \times 10^{6} \mathrm{~T}-47 \mathrm{D}$ cells were seeded in six-well plates and infected with the specific recombinant lentivirus (Lv-shCTSL or Lv-shCon) at a multiplicity of infection of 25 for $96 \mathrm{~h}$. Knockdown of endogenous CTSL was confirmed using western blot analysis with a monoclonal antibody (1:1,000; cat. no. TA809346; OriGene Technologies, Inc.), and infection efficiency was determined by counting the number of green fluorescent protein (GFP)-positive cells under a fluorescent microscope (magnification, $\mathrm{x} 400$ ).

Western blot analysis. The total cell proteins were extracted using RIPA buffer supplemented with a protease inhibitor cocktail (Roche Diagnostics). Total protein concentration was measured by BCA protein assay kit (Beyotime Institute of Biotechnology). Equal quantities of protein $(30 \mu \mathrm{g})$ were separated using 10\% SDS-PAGE and transferred onto PVDF membranes. The membranes were blocked in 5\% skimmed milk for $2 \mathrm{~h}$ at room temperature. Subsequently, the blots were probed with one of the following primary antibodies: Rabbit anti-CTSL (1:1,000; cat. no. TA809346; OriGene Technologies, Inc.); rabbit anti-CDK2AP1 (1:1,000; cat. no. 13060-2-AP; Proteintech Group, Inc.); or mouse anti-GAPDH (1:500,000; cat. no. HRP-60004; Proteintech Group, Inc.) overnight at $4^{\circ} \mathrm{C}$, followed by incubation with the secondary anti-rabbit horseradish peroxidase (HRP)-conjugated antibody (1:5,000; cat. no. SC-2054; Proteintech Group, Inc.) or anti-mouse HRP (1:5,000; cat. no. SC-2005; Santa Cruz Biotechnology, Inc.). The blots were visualized using an enhanced chemiluminescence reagent (Beyotime Institute of Biotechnology) and scanned using the Tanon 5200 (Tanon Science and Technology Co., Ltd.) and analyzed using the Image J software v1.48 (National Institutes of Health).

Glutathione S-transferase (GST) pull-down assay. Direct physical interactions between CTSL and CDK2-AP1 in eukaryotic cells were examined using a GST pull-down assay. A GST or GST-CDK2-AP1 fusion protein was expressed and purified as bait from Escherichia coli BL21/DE3 recombinants induced with isopropyl $\beta$-D-1-thiogalactopyranoside, whereas the GFP-CTSL fusion protein was overexpressed in $293 \mathrm{~T}$ cells as prey. A total of $5 \mathrm{ng}$ purified GST-labeled proteins were incubated with $25 \mu 1$ glutathione sepharose 4B beads for $3 \mathrm{~h}$ at $4^{\circ} \mathrm{C}$ and incubated with the cell lysate from the $293 \mathrm{~T}$ recombinants overnight at $4^{\circ} \mathrm{C}$. The bead-bound protein complexes were detected using western blotting (rabbit anti-GFP; 1:8,000; cat. no. 50430-2-AP; rabbit anti-GST; 1:6,000; cat. no. 10,000-0-AP; incubated overnight at $4^{\circ} \mathrm{C}$; antibodies from Proteintech Group, Inc.).
Table I. Patient clinicopathological characteristics.

\begin{tabular}{lc}
\hline Characteristics & $\begin{array}{c}\text { No. of patients }(\%) \\
(\mathrm{N}=249)\end{array}$ \\
\hline Age, years & \\
$\leq 50$ & $159(63.8)$ \\
$>50$ & $90(36.1)$ \\
Menstrual status & \\
Pre-menopausal & $160(64.2)$ \\
Post-menopausal & $88(35.3)$ \\
Missing & $1(0.4)$ \\
Stage & \\
I-II & $197(79.1)$ \\
III & $48(19.3)$ \\
Missing & $4(1.6)$ \\
Lymph node metastasis & \\
Positive & $126(50.6)$ \\
Negative & $122(49.0)$ \\
Missing & $1(0.4)$ \\
Estrogen receptor & \\
Positive & $140(56.2)$ \\
Negative & $104(41.8)$ \\
Missing & $5(2.0)$ \\
Progesterone receptor & \\
Positive & $131(52.6)$ \\
Negative & $112(45.0)$ \\
Missing & $6(2.4)$ \\
Human endothelial growth factor & \\
receptor 2 expression & \\
Positive & \\
Negative & \\
Missing & $16(30.5)$ \\
\hline & $1(0.4)$ \\
\hline
\end{tabular}

Proliferation assay. The proliferation or viability of the tumor cells with or without CTSL-knockdown was assessed using an MTT assay (Sigma-Aldrich; Merck KGaA). Briefly, T-47D cells transfected with either Lv-shCon or Lv-shCTSL were plated into a 96-well plate at a density of $3 \times 10^{3}$ cells/well and incubated with $20 \mu 1$ MTT $(5 \mathrm{mg} / \mathrm{ml})$ at $37^{\circ} \mathrm{C}$ for a further $4 \mathrm{~h}$ at the indicated time-points (day 1, 2, 3, 4 and 5). Subsequently, the formazan crystals were dissolved with $100 \mu \mathrm{l}$ acidic isopropanol (10\% SDS, 5\% isopropanol and $0.01 \mathrm{~mol} / 1 \mathrm{HCl}$ ) and the absorbance was measured at $570 \mathrm{~nm}$ using a microplate spectrophotometer.

Colony formation assay. Lentivirus-infected T-47D cells were trypsinized and seeded into a six-well plate at a density of $1 \times 10^{3}$ cells/well and incubated for 14 days until colonies were visible by eye. The samples were carefully washed twice with PBS, fixed with $4 \%$ paraformaldehyde for $30 \mathrm{~min}$ and stained with crystal violet for $15 \mathrm{~min}$ at room temperature. The number of colonies in each group was visually counted manually and the average values were calculated. 
Flow cytometric analysis of apoptosis and cell cycle distribution. Flow cytometry was used to determine the proportion of apoptotic cells and the cell cycle distribution in breast cancer cells following CTSL knockdown. T-47D cells infected with $\mathrm{Lv}$-shCon or Lv-shCTSL were cultured to $80 \%$ confluency, harvested and washed twice with PBS. For cell cycle analysis, the cells were fixed and permeabilized with $-20^{\circ} \mathrm{C}$ $70 \%$ ethanol for $12 \mathrm{~h}$ prior to treating with DNase-free RNase and stained with propidium iodide (both Sigma-Aldrich; Merck $\mathrm{KGaA}$ ) for $30 \mathrm{~min}$ at $4^{\circ} \mathrm{C}$. For the analysis of apoptosis, the cells were resuspended in binding buffer containing Annexin V-fluorescein isothiocyanate and 7-AAD for $15 \mathrm{~min}$ in the dark according to the manufacturer's protocol (Annexin APC/V-7-AAD apoptosis detection kit, Nanjing KeyGen Biotech Co., Ltd.). The stained cells were subsequently subjected to flow cytometry analysis using a FACScalibur flow cytometer (BD Biosciences). The data was analyzed using FlowJo v7.6 software (FlowJo LLC).

Statistical analysis. Data were analyzed using either paired or unpaired Student's t-test to identify differences between two groups. One-way or two-way ANOVA with Dunnett's post hoc test to compare multiple groups using GraphPad prism v5.0 software (GraphPad Software, Inc.). $\mathrm{P}<0.05$ was considered to indicate a statistically significant difference. mRNA expression levels of CTSL in cancer and normal clinical specimens in six databases were compared using paired or unpaired Student's t-test. The prognostic value of CTSL expression in breast cancer was determined using Kaplan-Meier analysis of overall survival or PFS using PROGgene and compared using the log rank test, a tool that assesses the effects of genes on survival in patients with cancer (33). The covariates and cumulative PFS rates were calculated using a Cox-proportional hazard model with SPSS version 18.0 (SPSS, Inc.).

\section{Results}

CTSL directly interacts with CDK2-AP1. Our previous study demonstrated that CDK2-AP1 serves as a tumor suppressor in breast cancer in vitro and in vivo (34). To identify the proteins interacting with CDK2-AP1, Y2H screening was performed. Sequencing from the positive colonies identified 13 potential putative CDK2-AP1-interacting proteins, and the interaction networks were reconstructed using Cytoscape and STRING (Fig. 1A and B). To determine whether CTSL interacted with CDK2-AP1, a GST pull-down assay was performed with GST-CDK2-AP1 fusion protein as bait to capture GFP-CTSL from lysates of engineered 293T cells. The results demonstrated that GFP-CTSL could be pulled down from the cell lysates by GST-CDK2-AP1, but not by GST, suggesting a direct interaction between CTSL and CDK2-AP1 (Fig. 1C and D). Western blot analysis revealed that in cell lines exhibiting high expression of CTSL, CDK2-AP1 expression was low, whereas low level of CTSL corresponded to high CDK2-AP1 expression (Fig. 1E-G), suggesting that a balance between CTSL and CDK2-AP1 may be involved in the regulation of tumorigenesis.

CTSL is upregulated and associated with poor prognosis in patients with breast cancer. Immunohistochemistry was performed to determine CTSL expression levels in patients with breast cancer, and PFS analysis was performed to determine its prognostic value (Fig. 2A and B). CTSL was upregulated in breast cancer tissues compared with adjacent normal tissues (Fig. 2C and D). For PFS analysis, the median follow-up time was 7.59 years; CTSL expression levels $(\mathrm{P}=0.006)$, menstrual $(\mathrm{P}=0.007)$, lymph node metastasis $(\mathrm{P}<0.001), \mathrm{ER}(\mathrm{P}=0.004)$ and $\mathrm{PR}(\mathrm{P}=0.014)$ statuses were significantly associated with PFS in the univariate Cox-proportional hazard model (Table II). Patients with low CTSL expression levels exhibited a higher PFS rate (Fig. 2B). The parameters significantly associated with PFS were analyzed using multivariate Cox regression analysis. By adjusting menstrual status, lymph node metastasis, ER and PR status as covariates, CTSL expression level was not significantly associated with PFS in patients with breast cancer (hazard ratio, $0.61 ; 95 \%$ confidence interval, 0.35-1.09; $\mathrm{P}=0.096$; Table II).

To further investigate the role of CTSL in breast cancer, six independent microarray datasets from the ONCOMINE database were analyzed, which exhibited statistically higher CTSL expression levels in the majority of breast cancer tissues compared with adjacent non-cancerous tissues (Fig. 3A). In addition, increased expression of CTSL was associated with histological grade and clinical stage of breast cancer (Fig. 3B). To further investigate the prognostic significance of CTSL expression in patients with breast cancer, Kaplan-Meier analysis was performed using the PROGgene database (33). Patients with high CTSL expression exhibited worse overall survival compared with patients with low CTSL expression levels, suggesting that CTSL served as a promoting factor during the tumorigenesis and malignant progression of breast cancer, which may be regarded as a valuable hallmark for prognosis.

CTSL knockdown reduces the progression of breast cancer cells in vitro. As upregulation of CTSL was significantly associated with the progression and poor prognosis in breast cancer, the pathological functions of CTSL were studied in vitro. T-47D cells were selected due to high expression levels of CTSL (Fig. 1D). Endogenous CTSL was knocked down using the specific Lv-shCTSL and CDK2-AP1 was increased in CTSL-downregulated cells (Fig. 4A). An MTT assay demonstrated that the proliferation of T-47D cells transfected with shCTSL was decreased significantly compared with those transfected with shCon (Fig. 4B). Colony formation of T-47D cells was significantly decreased following CTSL downregulation compared with the negative control (Fig. 4C), suggesting that CTSL may contribute to the proliferation and colony formation of breast cancer cells. In addition, flow cytometry revealed that knockdown of CTSL expression significantly induced G0/G1-phase arrest in T-47D cells and increased the apoptotic index compared with the control cells (Fig. 4D and E), suggesting that CTSL knockdown may decelerate the progression of breast cancer by arresting the cell cycle and inducing apoptosis.

\section{Discussion}

Accumulating evidence has demonstrated that proteolytic enzymes serve a contributing role in cancer 
A

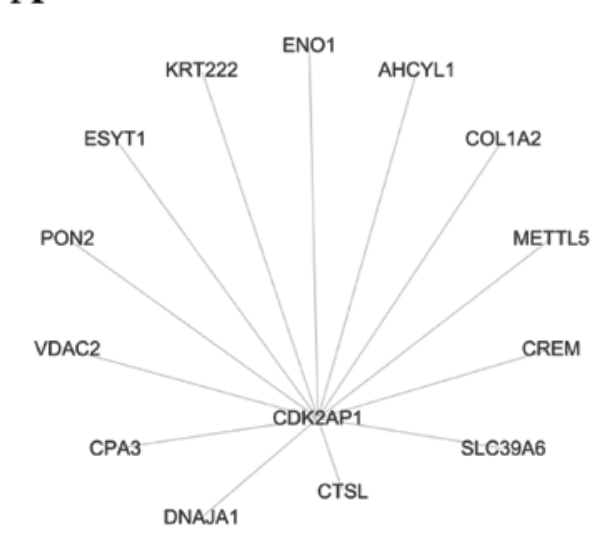

B

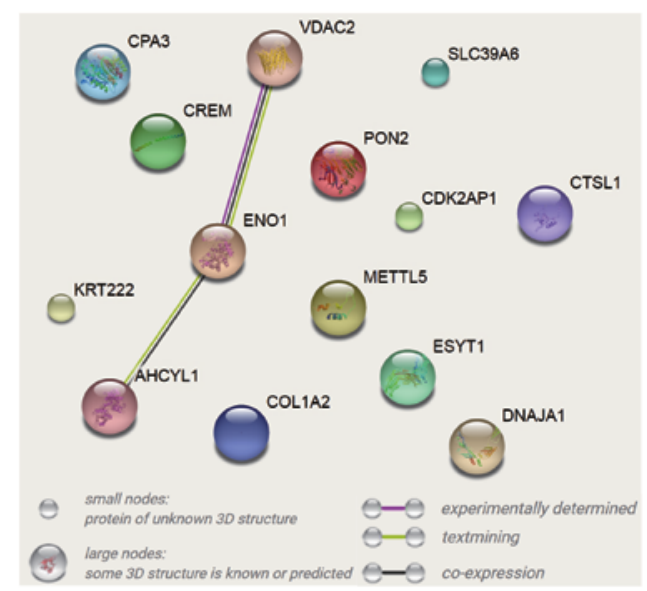

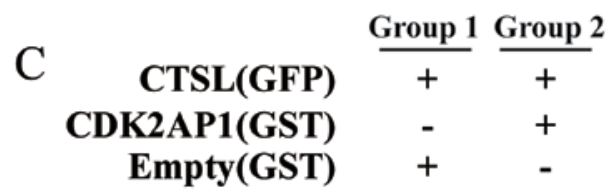

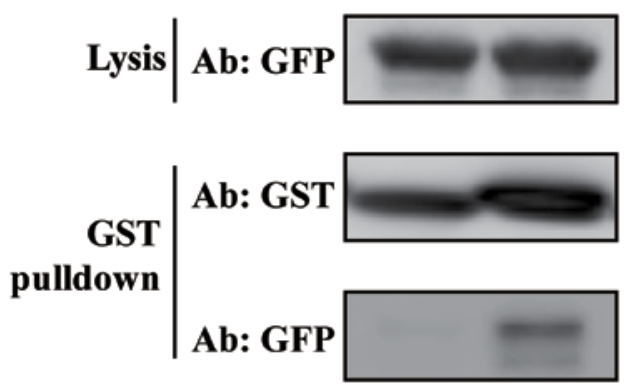

F

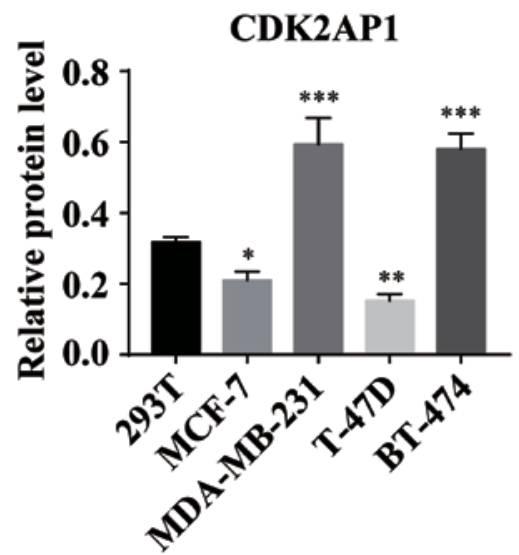

D $\overline{\mathrm{d}}_{\mathrm{d}}^{\mathrm{d}} 181.5-$ Group 1

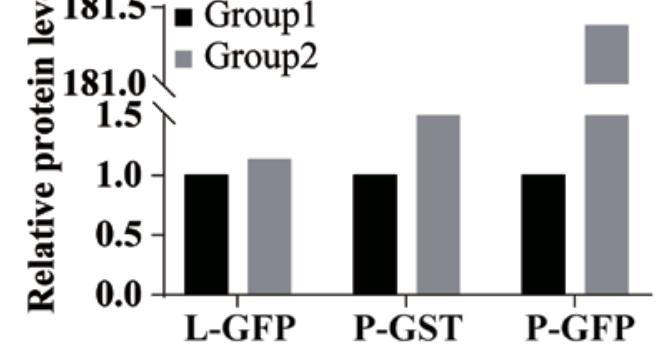

$\mathrm{E}$

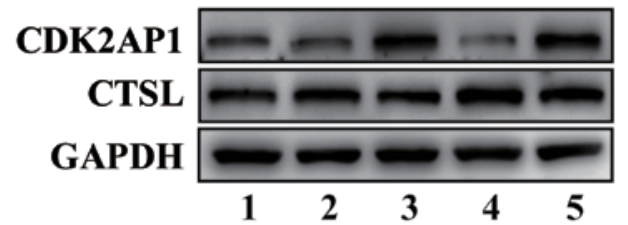

G

CTSL

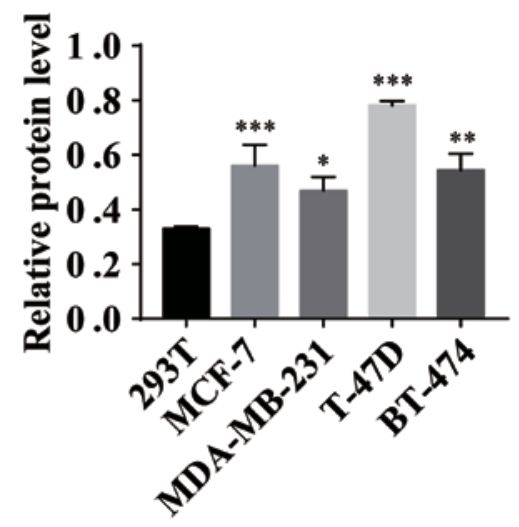

Figure 1.CTSL directly interacts with CDK-2AP1.(A) Protein interaction map identified by yeast two-hybrid screening indicated putative CDK2-AP1-interacting proteins using Cytoscape. (B) Protein-protein interaction networks of the indicated candidates were constructed using Search Tool for the Retrieval of Interacting Genes/Proteins database. (C) GST pull-down assay was performed in 293T cells to identify the interaction of CTSL and CDK2-AP1. (D) The densitometry analysis of the protein bands of GST pull-down assay. (E) Western blot analysis was used to determine the endogenous expression of CDK2AP1 and CTSL in breast cancer cell lines. 1, 2, 3, 4 and 5 represents 293T, MCF-7, MDA-MB-231, T-47D and BT-474, respectively. (F) CDK2-AP1 protein expression in breast cancer cell lines. (G) CTSL protein expression in breast cancer cell lines. Statistical analyses were performed using one-way ANOVA with Dunnett's multiple comparisons test: ${ }^{*} \mathrm{P}<0.05$ and ${ }^{* *} \mathrm{P}<0.01$, ${ }^{* * *} \mathrm{P}<0.001$ vs. 293T. CTSL, Cathepsin L; CDK2-AP1, cyclin-dependent kinase 2-associated protein 1; GST, glutathione S-transferase; GFP, green fluorescent protein.

metastasis $(35,36)$. Proteases are involved in several stages of tumorigenesis or malignant progression, including proteolytic activation of latent growth factors and pro-angiogenic factors, degradation of the extracellular and interstitial matrices, as well as intravasation or extravasation across the capillary/lymphatic system $(37,38)$. In light of the crucial functions of proteases in tumor development and progression, numerous inhibitors have been developed 
Table II. Univariate and multivariate Cox regression analyses of CTSL expression and PFS in patients with breast cancer.

\begin{tabular}{|c|c|c|c|c|}
\hline \multirow[b]{2}{*}{ Variables } & \multicolumn{4}{|c|}{ PFS } \\
\hline & $\begin{array}{l}\text { Univariate HR } \\
(95 \% \mathrm{CI})\end{array}$ & P-value & $\begin{array}{c}\text { Multivariate HR } \\
(95 \% \mathrm{CI})\end{array}$ & P-value \\
\hline $\operatorname{Age}(\leq 50$ vs. $>50)$ & $0.65(0.38-1.09)$ & 0.104 & & \\
\hline Menstrual status (pre-menopausal vs. post-menopausal) & $2.05(1.21-3.46)$ & $0.007^{\mathrm{a}}$ & $2.36(1.36-4.12)$ & $0.002^{\mathrm{a}}$ \\
\hline Stage (I-II vs. III) & $1.06(0.52-2.18)$ & 0.862 & & \\
\hline Lymph node metastasis (positive vs. negative) & $0.24(0.13-0.46)$ & $<0.001^{\mathrm{a}}$ & $0.21(0.11-0.40)$ & $<0.001^{\mathrm{a}}$ \\
\hline ER status (negative vs. positive) & $2.21(1.29-3.79)$ & $0.004^{\mathrm{a}}$ & $2.51(1.32-4.80)$ & $0.005^{\mathrm{a}}$ \\
\hline PR status (negative vs. positive) & $1.96(1.15-3.38)$ & $0.014^{\mathrm{a}}$ & $0.93(0.48-1.80)$ & 0.831 \\
\hline HER-2 status (negative vs. positive) & $0.83(0.47-1.46)$ & 0.512 & & \\
\hline CTSL expression (high vs. low) & $0.47(0.27-0.80)$ & $0.006^{\mathrm{a}}$ & $0.61(0.35-1.09)$ & 0.096 \\
\hline
\end{tabular}

${ }^{a} \mathrm{P}<0.05$. PFS, progression-free survival; ER, estrogen receptor; PR, progesterone receptor; HER-2, human endothelial growth factor receptor 2; $\mathrm{HR}$, hazard ratio; CI, confidence interval.
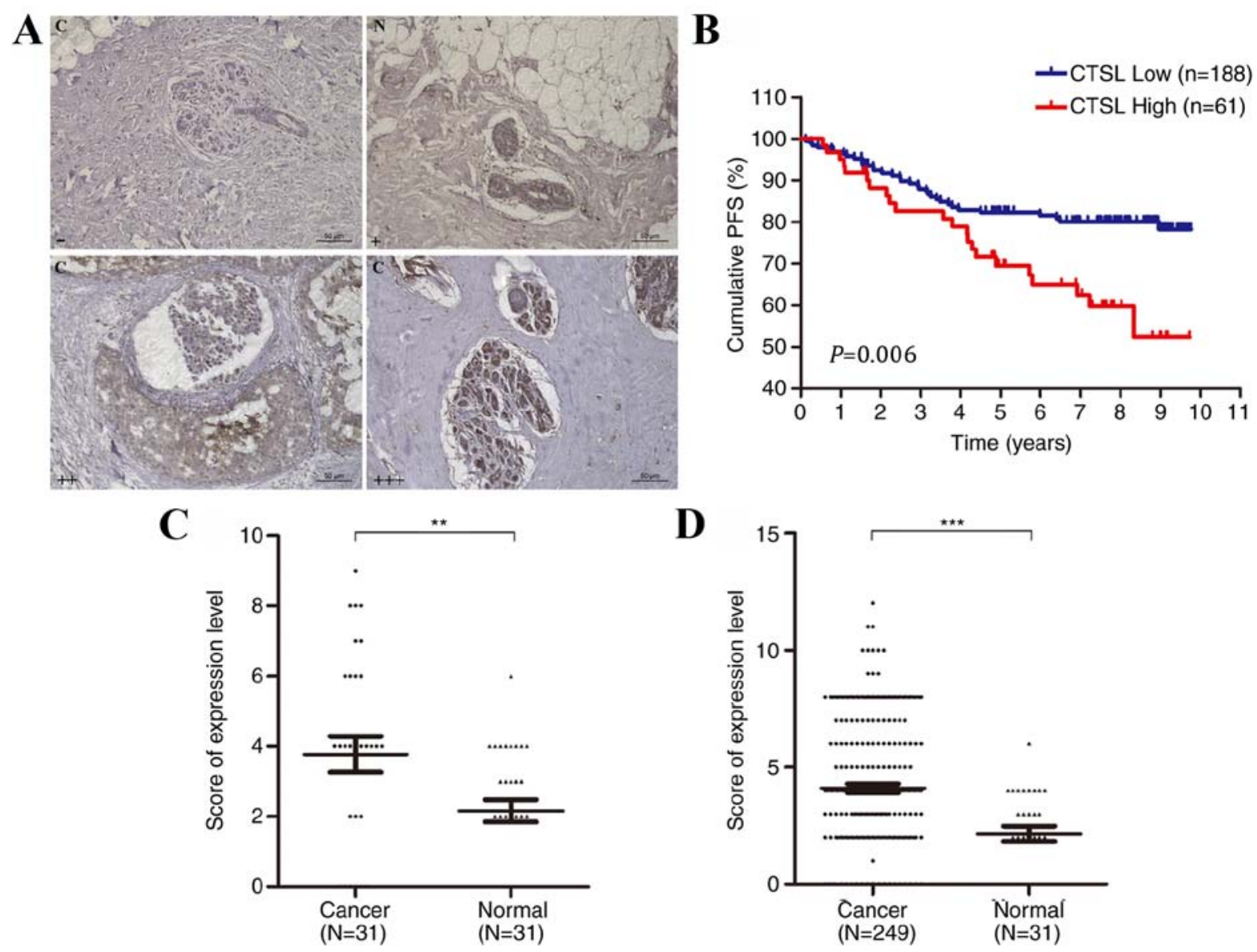

Figure 2. CTSL is upregulated and associated with poor PFS in patients with breast cancer. (A) Representative immunohistochemistry staining of CTSL expression in human breast cancer and adjacent normal tissues. C, Cancer; N, Normal; -, negative; +, poor positive; ++, moderate positive; +++, strong positive. (B) CTSL expression levels in 31 paired breast cancer tissues and adjacent normal tissues. (C) CTSL expression level in 249 breast cancer tissues and 31 adjacent normal tissues. (D) PFS analysis of patients with breast cancer with high and low CTSL expression levels. Kaplan-Meier analysis was used to determine the prognostic significance of CTSL expression in patients with breast cancer. Statistical significance between groups was calculated using either paired or unpaired Student's t-test: ${ }^{* *} \mathrm{P}<0.01$ and ${ }^{* * *} \mathrm{P}<0.001$. CTSL, Cathepsin L; PFS, progression-free survival.

and applied in clinical trials, such as MPP inhibitors. A number of protease-targeted drugs have been successful, although adverse side effects hinder their application in oncotherapy (39). Therefore, it is important to identify novel potential proteolytic targets and antagonists to impair tumor metastasis and progression. 


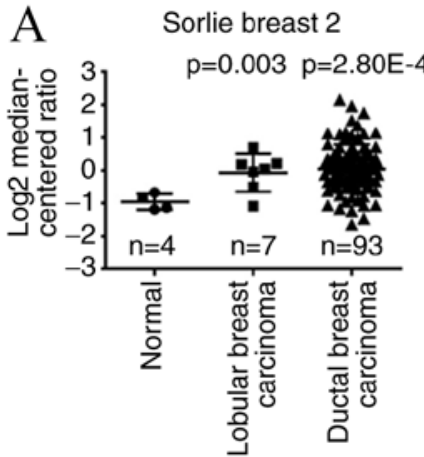

Zhao breast

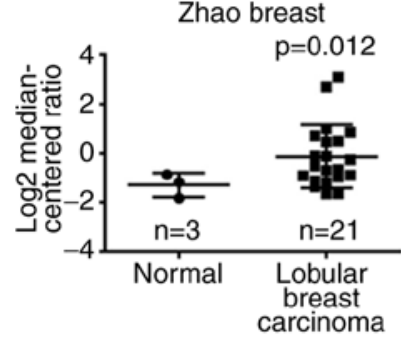

C GSE9893
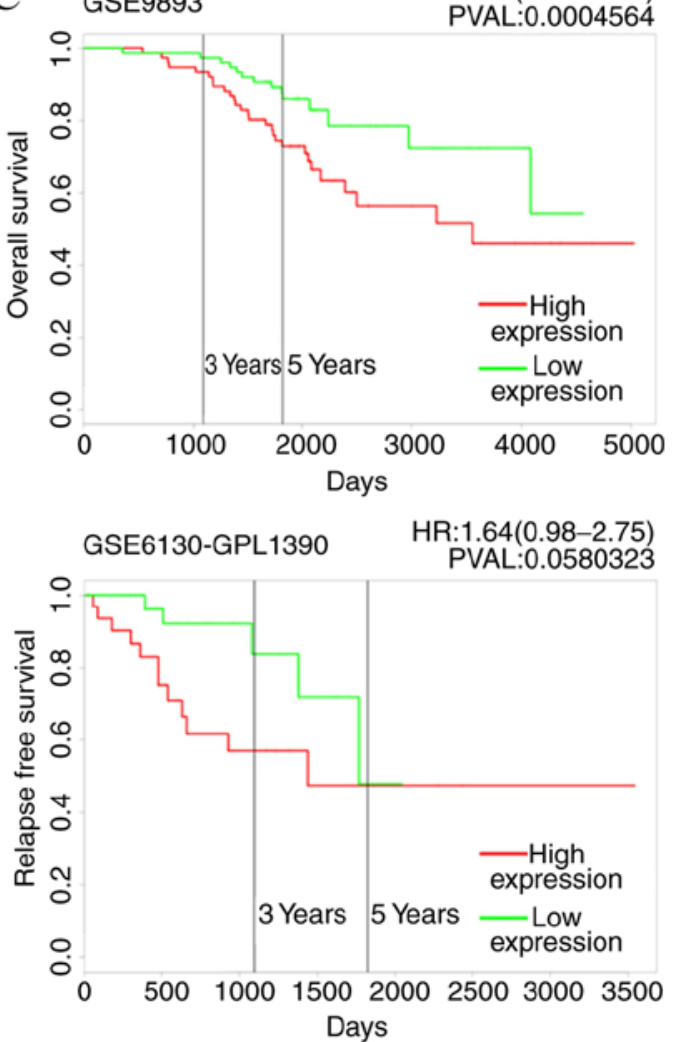

Perou breast

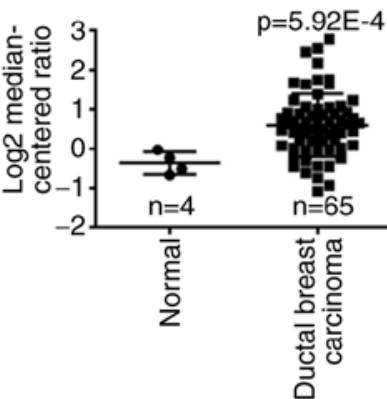

Richardson breast 2

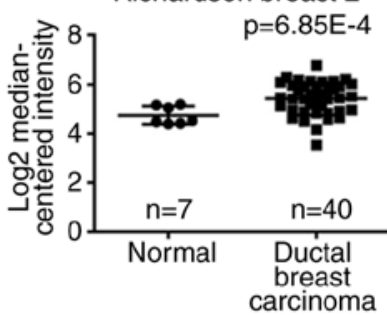

B
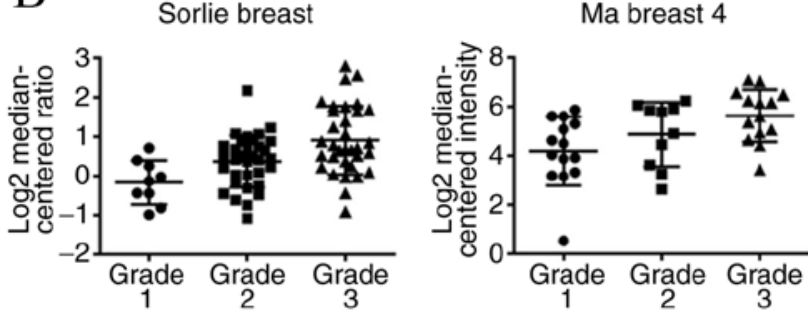

Ma breast 4
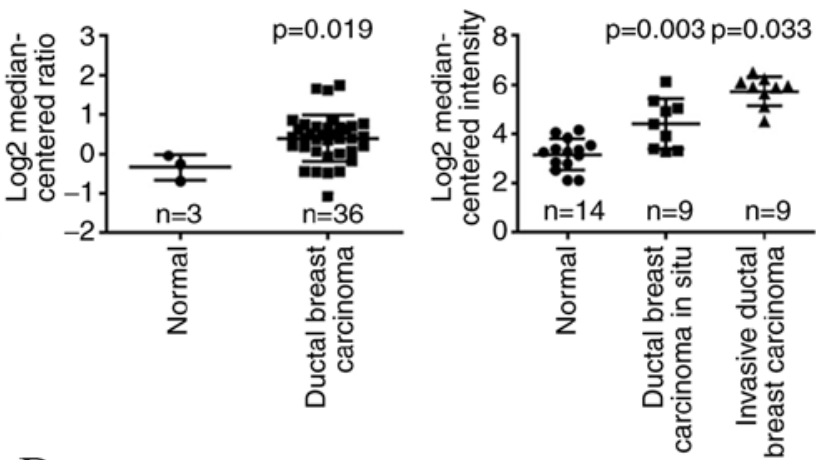

Ma breast 4
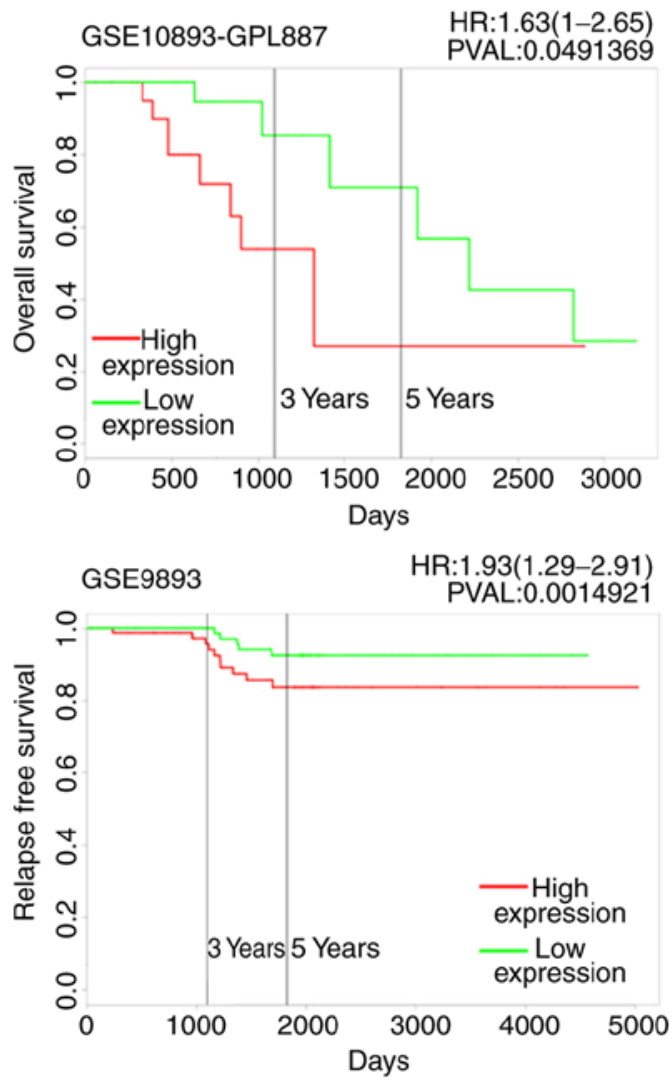

Figure 3. Upregulation of CTSL is associated with poor prognosis in patients with breast cancer. (A and B) Oncomine analysis of CTSL expression profiles in human breast cancer. The following cut-off criteria was used: P-value threshold was set at 0.01 to remove data with $\mathrm{P}>0.01$ and the fold-change was defined as 2 to exclude those data with CTSL expression fold-change <2. (C) Kaplan-Meier analysis of overall and relapse-free survival in the patients with breast cancer. The association between CTSL expression and survival was analyzed in GSE9893, GSE10893-GPL887 and GSE6130-GPL1390 data sets from Gene Expression Omnibus using the PROGgene database. Data were analyzed using Student's t-test between the two groups or one-way ANOVA among three groups and were expressed as the mean \pm standard deviation. CTSL, Cathepsin L.

In the present study, CTSL was identified as a potential target for treating breast cancer. CTSL is a member of the papain superfamily of cysteine proteases, is overexpressed in multiple cancers and associated with cancer metastasis (40-42), and perceived as a potential facilitator of tumorigenesis and neoplastic progression (18,43-45). For example, CTSL ablation led to a significant reduction in MDA-MB-231 tumor cell-induced angiogenesis in vitro and in vivo by affecting cell cycle-associated genes, including cyclin D1-D3, E2, A2, B2 and $\mathrm{H}$ (15). In addition, CTSL blocked TGF- $\beta$-induced cell migration 
$\mathbf{A}$

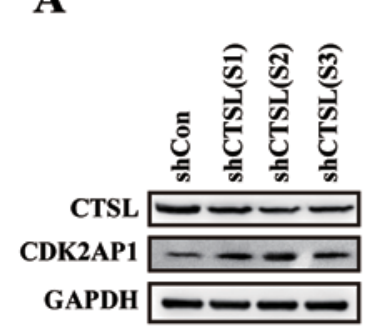

$\mathbf{D}$

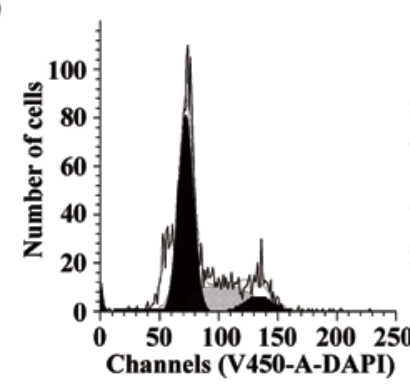

$\mathbf{E}$
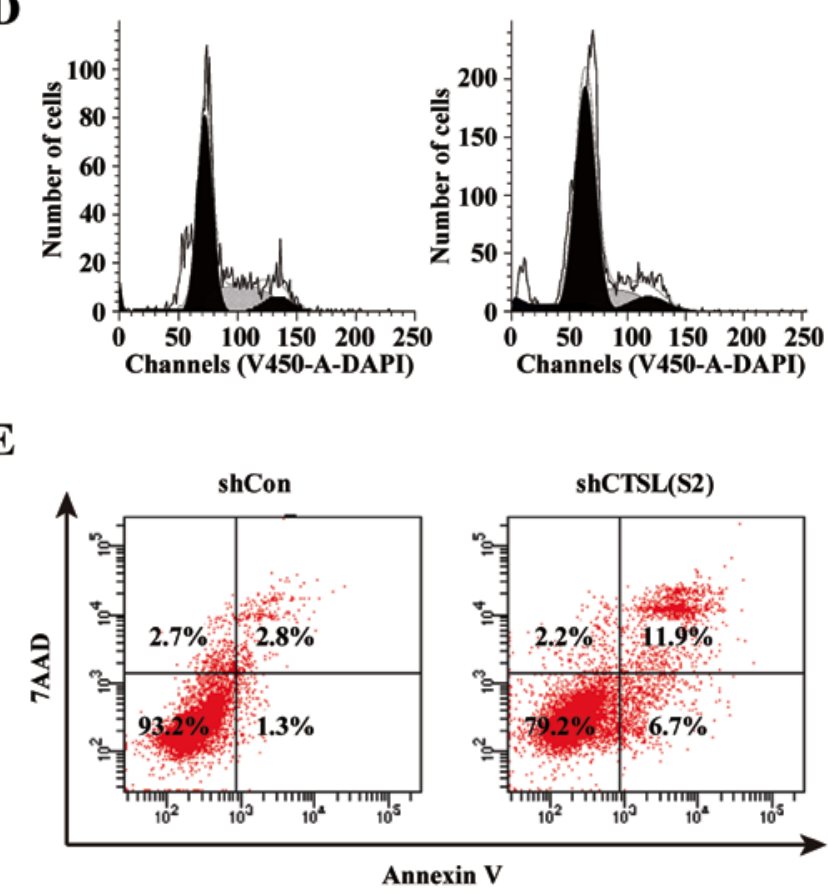

B
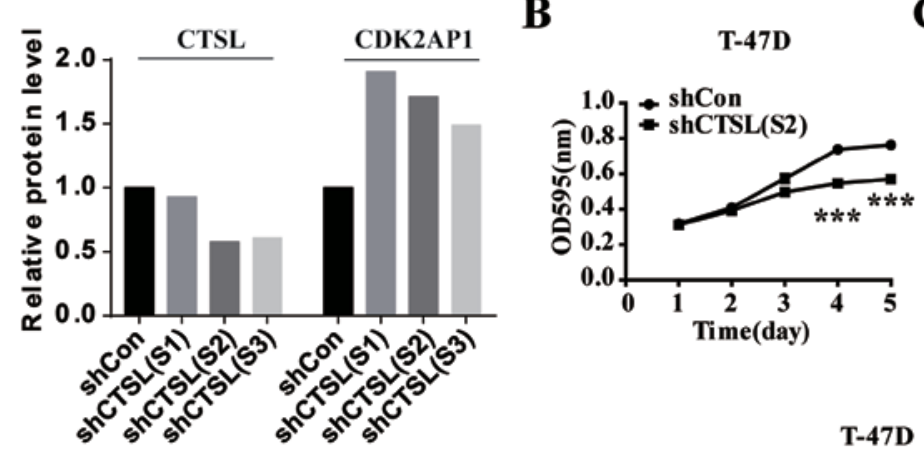

C

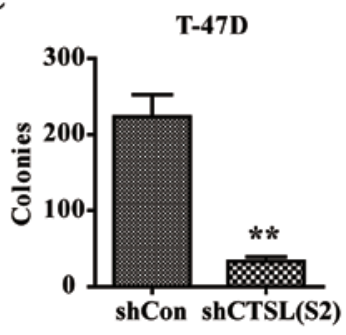

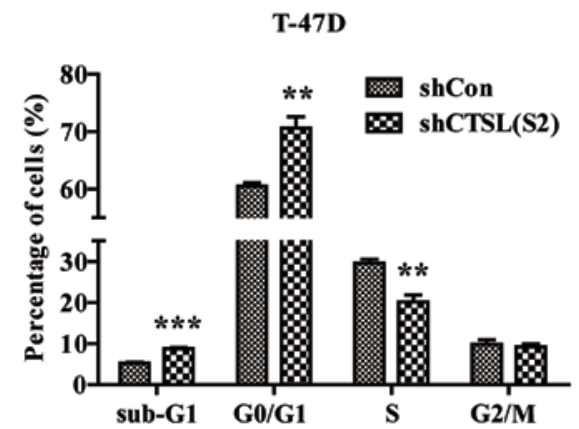

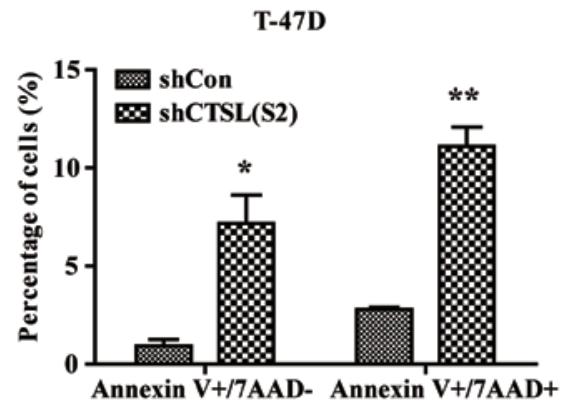

Figure 4. CTSL knockdown suppresses the proliferation and colony formation and induces G0/G1-phase arrest and apoptosis in T-47D cells. (A) Endogenous expression of CTSL was downregulated by the specific RNA interference lentivirus as determined using western blot analysis. (B) Proliferation was detected using an MTT assay and the results showed proliferation of T-47D cells was inhibited after knockdown of CTSL expression. (C) Colony formation assay with and without Lv-shCTSL infection revealed that inhibition of CTSL decreased the colony formation ability in T-47D cells. (D) Cell cycle distribution was determined using flow cytometric analysis in T-47D cells after CTSL knockdown (left panel). The percentage of cells in the $\mathrm{G}_{0} / \mathrm{G}_{1}, \mathrm{~S}$, and $\mathrm{G}_{2} / \mathrm{M}_{\text {phases }}$ was calculated (right panel). (E) T-47D cells transfected with shCTSL(S2) or shCon were stained and analyzed using flow cytometry. The percentage of apoptotic cells is presented. Data are presented as the mean \pm standard deviation of three independent experiments. Data were analyzed using Student's $t$-test for colony formation assay and two-way ANOVA with Dunnett's multiple comparisons test for the proliferation and flow cytometry assays: ${ }^{*} \mathrm{P}<0.05$, ${ }^{* *} \mathrm{P}<0.01$ and ${ }^{* * *} \mathrm{P}<0.001$ vs. shCon. CTSL, Cathepsin L; sh, short hairpin; OD, optical density; CDK2AP1, cyclin-dependent kinase 2-associated protein 1.

via the PI3K-AKT and Wnt signaling pathways in A549 and MCF-7 cell lines (46). Qin et al (47) demonstrated that CTSL affected proliferation of breast cancer; downregulation of CTSL significantly inhibited the proliferation of MCF-7 cells, which was similar to the results of the present study.

As previously reported (34), CDK2-AP1 serves as a tumor suppressor in breast cancer in vitro and in vivo. In the present study, Y2H screening further revealed 13 putative CDK2-AP1-interacting proteins, including CTSL; a direct physical interaction between CDK2-AP1 and CTSL was demonstrated using a GST pull-down assay. Analysis using the Oncomine and PROGgene databases revealed increased expression of CTSL in breast cancer tissues compared with normal tissues, and the expression profile of CTSL in tumor tissues exhibited a negative association with clinical outcomes and overall survival, suggesting a potential role for CTSL as a drug target in the clinical oncotherapy of breast cancer.
To delineate how CTSL functioned in breast cancer, the endogenous expression pattern of CTSL in a range of established breast cancer cell lines was initially determined. In agreement with the bioinformatics analysis, CTSL was upregulated in breast cancer cells compared with 293T cells and exhibited an inverse association with CDK2-AP1 expression. In addition, knockdown of CTSL in T-47D cells induced by lentiviral shRNA notably suppressed cell proliferation and colony formation in vitro, and T-47D cells exhibited G0/G1-phase arrest and increased apoptosis when CTSL was knocked down compared with the negative control, which was in agreement with our previous study (34). Knockdown of CTSL may thus interrupt the cell cycle and promote apoptosis. In addition, as T-47D is invasive, whether CTSL may affect the migration and invasion of breast cancer requires further study.

As CDK2-AP1 functions as a specific negative regulator for cyclin-dependent kinase 2 (CDK2) and CDK2 is a checkpoint 
in the cellular G1/S-phase $(48,49)$, CTSL may be an upstream negative regulator of CDK2-AP1 that proteolytically inactivates CDK2-AP1 and weakens or reverses its inhibition of CDK2. This mechanism may be explained by the observation that CTSL was occasionally detected in normal tissues, but upregulated in the majority of breast cancer tissues associated with malignant progression and poor prognosis. Additional studies are required to validate this hypothesis.

In conclusion, results of the present study demonstrated that CTSL may act as a tumor promoter by interfering with the tumor suppressor CDK2-AP1, leading to the progression of breast cancer. These findings suggest a novel potential drug target for the treatment of breast cancer.

\section{Acknowledgements}

Not applicable.

\section{Funding}

The present study was supported The National Natural Science Foundation of China (grant nos. 81572612, 81803640 and 81372842), the Hunan Provincial Natural Science Foundation (grant no. 2015JJ2183), Youth Science Foundation of Xiangya Hospital, Central South University (grant no. 2017Q02), The National Key Clinical Specialist Construction Programs of China, the Research Innovation Program for Graduate Students of Central South University (grant no. 2018zzts912) and The Program of China Scholarships Council (grant no. 201706370123 to ZW).

\section{Availability of data and materials}

The datasets used and analyzed during the present study are available from the corresponding author on reasonable request.

\section{Authors' contributions}

ZW, ZX, LQS and WBZ conceived and designed the experiments. ZW and TZ performed the experiments, ZW and JC analyzed the data. MZZ, JH, KSW, and LL contributed to the collection of clinical samples, performing experiments and data analysis. ZW wrote the manuscript. ZW, TZ, LQS and WBZ reviewed and edited the manuscript. All authors read and approved the final manuscript.

\section{Ethics approval and consent to participate}

The study was approved by The Ethical Review Committee of Xiangya Hospital, Central South University (Changsha, China). All patients provided written informed consent.

\section{Patient consent for publication}

Not applicable.

\section{Competing interests}

The authors declare that they have no competing interests.

\section{References}

1. Siegel RL, Miller KD and Jemal A: Cancer statistics, 2018. CA Cancer J Clin 68: 7-30, 2018.

2. Gansler T, Ganz PA, Grant M, Greene FL, Johnstone P, Mahoney M, Newman LA, Oh WK, Thomas CR Jr, Thun MJ, et al: Sixty years of CA: A cancer journal for clinicians. CA Cancer J Clin 60: 345-350, 2010.

3. DeSantis CE, Fedewa SA, Goding Sauer A, Kramer JL, Smith RA and Jemal A: Breast cancer statistics, 2015: Convergence of incidence rates between black and white women. CA Cancer J Clin 66: 31-42, 2016.

4. He M, Guo Q and Hu G: Reversed urban-rural differences in breast cancer mortality (China, 2002-2008). Breast Cancer Res Treat 126: 231-234, 2011.

5. Mahamodhossen YA, Liu W and Rong-Rong Z: Triple-negative breast cancer: New perspectives for novel therapies. Med Oncol 30: 653, 2013.

6. Bilal E, Dutkowski J, Guinney J, Jang IS, Logsdon BA, Pandey G, Sauerwine BA, Shimoni Y, Moen Vollan HK, Mecham BH, et al: Improving breast cancer survival analysis through competition-based multidimensional modeling. PLoS Comput Biol 9: e1003047, 2013.

7. Freedman GM and Fowble BL: Local recurrence after mastectomy or breast-conserving surgery and radiation. Oncology (Williston Park) 14: 1561-1581, 2000.

8. Duffy MJ: Biochemical markers in breast cancer: Which ones are clinically useful? Clin Biochem 34: 347-352, 2001.

9. Mohammadzadeh F, Mosayebi G, Montazeri V, Darabi M, Fayezi S, Shaaker M, Rahmati M, Baradaran B, Mehdizadeh A and Darabi M: Fatty acid composition of tissue cultured breast carcinoma and the effect of stearoyl-coa desaturase 1 inhibition. J Breast Cancer 17: 136-142, 2014.

10. Guo Y, Wang P, Li X, Zhu S, Xu H, Li S, Deng H and Yuan L: Identifying a BRCA2 c.5722_5723del mutation in a Han-Chinese family with breast cancer. Biosci Rep 39: 2019.

11. Rawlings ND, Barrett AJ and Bateman A: MEROPS: The database of proteolytic enzymes, their substrates and inhibitors. Nucleic Acids Res 40: D343-D350, 2012.

12. Kabel AM: Tumor markers of breast cancer: New prospectives. J Oncol Sci 3: 5-11, 2017.

13. Tan G, Liu Q, Tang X, Kang T, Li Y, Lu J, Zhao X and Tang F: Diagnostic values of serum cathepsin B and D in patients with nasopharyngeal carcinoma. BMC Cancer 16: 241, 2016.

14. Uhlman A, Folkers K, Liston J, Pancholi H and Hinton A: Effects of vacuolar H+-ATPase inhibition on activation of cathepsin B and Cathepsin L secreted from MDA-MB231 breast cancer cells. Cancer Microenviron 10: 49-56, 2017.

15. Sudhan DR, Rabaglino MB, Wood CE and Siemann DW: Cathepsin L in tumor angiogenesis and its therapeutic intervention by the small molecule inhibitor KGP94. Clin Exp Metastasis 33: 461-473, 2016

16. Liu Y, Li X, Peng D, Tan Z, Liu H, Qing Y, Xue Y and Shi GP: Usefulness of serum Cathepsin $\mathrm{L}$ as an independent biomarker in patients with coronary heart disease. Am J Cardiol 103: 476-481, 2009.

17. Sui H, Shi C, Yan Z and Wu M: Overexpression of Cathepsin L is associated with chemoresistance and invasion of epithelial ovarian cancer. Oncotarget 7: 45995-46001, 2016.

18. Sudhan DR and Siemann DW: Cathepsin L targeting in cancer treatment. Pharmacol Ther 155: 105-116, 2015.

19. Edge SB and Compton CC: The American joint committee on cancer: The 7th edition of the AJCC cancer staging manual and the future of TNM. Ann Surg Oncol 17: 1471-1474, 2010.

20. World Medical Association: World Medical Association declaration of helsinki: Ethical principles for medical research involving human subjects. JAMA 310: 2191-2194, 2013.

21. Wang Z, Chen J, Zhong MZ, Huang J, Hu YP, Feng DY, Zhou ZJ, Luo X, Liu ZQ, Jiang WZ and Zhou WB: Overexpression of ANLN contributed to poor prognosis of anthracycline-based chemotherapy in breast cancer patients. Cancer Chemother Pharmacol 79: 535-543, 2017.

22. Sorlie T, Perou CM, Tibshirani R, Aas T, Geisler S, Johnsen H, Hastie T, Eisen MB, van de Rijn M, Jeffrey SS, et al: Gene expression patterns of breast carcinomas distinguish tumor subclasses with clinical implications. Proc Natl Acad Sci USA 98: 10869-10874, 2001. 
23. Sorlie T, Tibshirani R, Parker J, Hastie T, Marron JS, Nobel A, Deng S, Johnsen H, Pesich R, Geisler S, et al: Repeated observation of breast tumor subtypes in independent gene expression data sets. Prolc Natl Acad Sci USA 100: 8418-8423, 2003.

24. Perou CM, Sorlie T, Eisen MB, van de Rijn M, Jeffrey SS, Rees CA, Pollack JR, Ross DT, Johnsen H, Akslen LA, et al: Molecular portraits of human breast tumours. Nature 406: 747-752, 2000

25. Ma XJ, Dahiya S, Richardson E, Erlander M and Sgroi DC: Gene expression profiling of the tumor microenvironment during breast cancer progression. Breast Cancer Res 11: R7, 2009.

26. Zhao H, Langerod A, Ji Y, Nowels KW, Nesland JM, Tibshirani R, Bukholm IK, Kåresen R, Botstein D, Børresen-Dale AL and Jeffrey SS: Different gene expression patterns in invasive lobular and ductal carcinomas of the breast. Mol Biol Cell 15: 2523-2536, 2004.

27. Richardson AL, Wang ZC, De Nicolo A, Lu X, Brown M, Miron A, Liao X, Iglehart JD, Livingston DM and Ganesan S: X chromosomal abnormalities in basal-like human breast cancer. Cancer Cell 9: 121-132, 2006.

28. Weigman VJ, Chao HH, Shabalin AA, He X, Parker JS, Nordgard SH, Grushko T, Huo D, Nwachukwu C, Nobel A, et al: Basal-like breast cancer DNA copy number losses identify genes involved in genomic instability, response to therapy, and patient survival. Breast Cancer Res Treat 133: 865-880, 2012.

29. Mullins M, Perreard L, Quackenbush JF, Gauthier N, Bayer S, Ellis M, Parker J, Perou CM, Szabo A and Bernard PS: Agreement in breast cancer classification between microarray and quantitative reverse transcription PCR from fresh-frozen and formalin-fixed, paraffin-embedded tissues. Clin Chem 53: 1273-1279, 2007.

30. Chanrion $M$, Negre V, Fontaine $H$, Salvetat N, Bibeau $F$, Mac Grogan G, Mauriac L, Katsaros D, Molina F, Theillet C and Darbon JM: A gene expression signature that can predict the recurrence of tamoxifen-treated primary breast cancer. Clin Cancer Res 14: 1744-1752, 2008.

31. Shannon P, Markiel A, Ozier O, Baliga NS, Wang JT, Ramage D, Amin N, Schwikowski B and Ideker T: Cytoscape: A software environment for integrated models of biomolecular interaction networks. Genome Res 13: 2498-2504, 2003.

32. Szklarczyk D, Franceschini A, Wyder S, Forslund K, Heller D, Huerta-Cepas J, Simonovic M, Roth A, Santos A, Tsafou KP, et al: STRING v10: Protein-protein interaction networks, integrated over the tree of life. Nucleic Acids Res 43: D447-D452, 2015.

33. Goswami CP and Nakshatri H: PROGgeneV2: Enhancements on the existing database. BMC Cancer 14: 970, 2014.

34. Zhou W, Guan X, Wang L, Liao Y and Huang J: p12(CDK2-AP1) inhibits breast cancer cell proliferation and in vivo tumor growth. J Cancer Res Clin Oncol 138: 2085-2093, 2012.

35. Herszenyi L, Barabas L, Hritz I, Istvan G and Tulassay Z: Impact of proteolytic enzymes in colorectal cancer development and progression. World J Gastroenterol 20: 13246-13257, 2014.

36. Herszenyi L, Lakatos G, Hritz I, Varga MZ, Cierny G and Tulassay Z: The role of inflammation and proteinases in tumor progression. Dig Dis 30: 249-254, 2012.
37. Mason SD and Joyce JA: Proteolytic networks in cancer. Trends Cell Biol 21: 228-237, 2011.

38. Tan GJ, Peng ZK, Lu JP and Tang FQ: Cathepsins mediate tumor metastasis. World J Biol Chem 4: 91-101, 2013

39. Turk B: Targeting proteases: Successes, failures and future prospects. Nat Rev Drug Discov 5: 785-799, 2006.

40. Tholen M, Wolanski J, Stolze B, Chiabudini M, Gajda M, Bronsert P, Stickeler E, Rospert S and Reinheckel T: Stress-resistant translation of Cathepsin L mRNA in breast cancer progression. J Biol Chem 290: 15758-15769, 2015.

41. Sudhan DR, Pampo C, Rice L and Siemann DW: Cathepsin L inactivation leads to multimodal inhibition of prostate cancer cell dissemination in a preclinical bone metastasis model. Int J Cancer 138: 2665-2677, 2016.

42. Sigloch FC, Tholen M, Gomez-Auli A, Biniossek ML, Reinheckel $\mathrm{T}$ and Schilling O: Proteomic analysis of lung metastases in a murine breast cancer model reveals divergent influence of CTSB and CTSL overexpression. J Cancer 8: 4065-4074, 2017.

43. Cao Y, Liu X, Li Y, Lu Y, Zhong H, Jiang W, Chen AF, Billiar TR, Yuan $\mathrm{H}$ and Cai J: Cathepsin $\mathrm{L}$ activity correlates with proteinuria in chronic kidney disease in humans. Int Urol Nephrol 49: 1409-1417, 2017.

44. Fei Y, Xiong Y, Zhao Y, Wang W, Han M, Wang L, Tan C and Liang Z: Cathepsin L knockdown enhances curcumin-mediated inhibition of growth, migration, and invasion of glioma cells. Brain Res 1646: 580-588, 2016.

45. Han ML, Zhao YF, Tan CH, Xiong YJ, Wang WJ, Wu F, Fei Y, Wang L and Liang ZQ: Cathepsin L upregulation-induced EMT phenotype is associated with the acquisition of cisplatin or paclitaxel resistance in A549 cells. Acta Pharmacol Sin 37: 1606-1622, 2016.

46. Qingqing Z, Meiling H, Wenjuan W, Song Y, Chen G, Wang Z and Liang Z: Downregulation of Cathepsin L suppresses cancer invasion and migration by inhibiting transforming growth factor? $\beta$ ?mediated epithelial?mesenchymal transition. Oncol Rep 33: 1851-1859, 2015.

47. Qin G, Cai Y, Long J, Zeng H, Xu W, Li Y, Liu M, Zhang H, He ZL and Chen WG: Cathepsin L is involved in proliferation and invasion of breast cancer cells. Neoplasma 63: 30-36, 2016.

48. He X, Xiang H, Zong X, Yan X, Yu Y, Liu G, Zou D and Yang H: CDK2-AP1 inhibits growth of breast cancer cells by regulating cell cycle and increasing docetaxel sensitivity in vivo and in vitro. Cancer Cell Int 14: 130, 2014.

49. Chai J, Ju J, Zhang SW, Shen ZY, Liang L, Yang XM, Ma C, Ni QW and Sun MY: p12CDK2-AP1 interacts with CD82 to regulate the proliferation and survival of human oral squamous cell carcinoma cells. Oncol Rep 36: 737-744, 2016.

This work is licensed under a Creative Commons Attribution-NonCommercial-NoDerivatives 4.0 International (CC BY-NC-ND 4.0) License. 